\title{
A 3-Year Retrospective Study of Community-Acquired Pneumonia in Kuwait with Emphasis on Microbial Aetiology
}

\author{
Nasser Awadh-Behbehani ${ }^{a}$ Zuhair Bitar ${ }^{b}$ Fatima Al-Enezic \\ Sana Al-Mutairi ${ }^{a}$ Mohamed Al-Azemi ${ }^{d}$ Faisal Al-Sayeq ${ }^{a}$ \\ aDepartments of Medicine, Kuwait University, ${ }^{\mathrm{b}} \mathrm{Al}-\mathrm{Adan}$ Hospital, ${ }^{\mathrm{c}} \mathrm{Al}-\mathrm{Am}$ iri Hospital, and \\ dAl-Farwaniya Hospital, Kuwait
}

\section{Key Words}

Pneumonia, community-acquired .

Aetiology Adults $\cdot$ Kuwait

\begin{abstract}
Background: There has not been any published study on community-acquired pneumonia (CAP) in Kuwait. The aim of this study is to determine the clinical characteristics of adult patients with CAP as well as the microbial aetiology and the treatment of CAP. Methods: A retrospective study of all CAP cases admitted to the three acute care hospitals in Kuwait over a 3-year period, 19931995, was done. Results: There were 290 patients identified, mean age $41 \pm 19$ years, of which $57 \%$ were males. The patients were divided into three groups (mild, moderate and severe) according to standard published criteria: mild $27.5 \%$, moderate $61 \%$, and severe $11.5 \%$. The microbial aetiology was identified for only $15 \%$ of the total cases,
\end{abstract}

\begin{tabular}{ll}
\hline KARGER & (1) 1998 S. Karger AG, Basel \\
Fax +4161306 1234 34 1011-7571/00/0093-0214\$17.50/0 \\
$\begin{array}{l}\text { E-Mail karger@karger.ch } \\
\text { www.karger.com }\end{array}$ & $\begin{array}{l}\text { Accessible online at: } \\
\text { www.karger.com/journals/mpp }\end{array}$
\end{tabular}

$11.5 \%$ of the mild group, $14 \%$ of the moderate, and $22.5 \%$ of the severe. The most common organisms identified were Streptococcus pneumoniae 32\%, Mycoplasma pneumoniae 20\%, Haemophilus influenzae 14\%, Mycobacterium tuberculosis 11\%, gramnegative organisms other than Haemophilus species $11 \%$ and others $12 \%$. There were 243 (84\%) patients who received a single antibiotic as initial therapy while the rest received a combination of antibiotics. The most common antibiotics used were macrolide $28 \%$, ampicillin $24 \%$, a combination of antibiotic $17 \%$, third-generation cephalosporin 12\% and others $19 \%$. Conclusion: The rate of identification of microbial aetiology in CAP is poor in Kuwait. There are $27.5 \%$ of the patients who can be classified as mild and probably the majority of them can be treated as out-patients. Based on these results, we have started a prospective study to better evaluate the microbial aetiology of CAP in Kuwait.

Dr. Nasser Behbehani, Assistant Professor

Department of Medicine, Kuwait University

PO Box 24923

Safat 13110 (Kuwait)

Fax +965 5338907, E-Mail nasser_beh@hsc.kuniv.edu.kw 


\section{Introduction}

Pneumonia is a common problem worldwide. Recently, there has been a study on lower respiratory tract infections among children in Kuwait [1]. However, there is no published study on community-acquired pneumonia (CAP) among adults in Kuwait. It is well recognised that the microbial aetiology of CAP is diverse and relatively new pathogens such as Legionalla and Chlamydia are becoming more important $[2,3]$. The frequency of occurrence of these organisms versus the usual other organisms such as streptococcal pneumonia among patients with CAP in $\mathrm{Ku}-$ wait is not known. Furthermore, several national guidelines on the initial management of CAP have been published recently [4-6]. The initial management of CAP and choice of antibiotics used in Kuwait have not been described before. Therefore we conducted this study to describe the clinical characteristics of patients presenting to hospital with CAP as well as the microbial aetiology and the outcome of treatment of CAP in Kuwait.

\section{Subjects and Methods}

The study was conducted in three acute care hospitals (Al-Adan, Al-Amiri and Farwaniya hospitals) in Kuwait. The medical records of all patients admitted to the three hospitals over a 3 year-period (1993-1995) with the following admission diagnoses: pneumonia, chest infection, and bronchitis were identified. The inclusion criteria for the study were age $\geq 15$ years, presence of fever, cough, and purulent sputum or chest radiograph abnormality consistent with pneumonia. The chest radiograph abnormality that was considered as pneumonia was either a lobar or segmental consolidation or an interstitial abnormality coupled with other clinical features of pneumonia like fever, cough or purulent sputum. Patients who did not have definite radiological sign of pneumonia or patients whose chest radiograph abnormalities were proved to be due to causes other than pneumonia were excluded from the study. Standardised data collection form was used for all the hospitals. Information on demographics, clinical presentations, laboratory investigations, microbial aetiology, treatment and outcome was collected. The patients were classified into mild, moderate and severe cases according to the following criteria [6]: mild pneumonia: age $<60$ and absence of any co-morbid conditions; moderate pneumonia: age $\geq 60$, fever $\geq 38.3^{\circ} \mathrm{C}$ and or presence of any co-morbid conditions; severe pneumonia: presence of any of the following signs regardless of age or co-morbid conditions: (1) admission to ICU, (2) shock, systolic BP $<90$ or diastolic BP $<60$, (3) altered mental status, (4) respiratory rate $\geq 30$ breaths per minute, (5) severe hypoxaemia with arterial partial pressure of $\mathrm{O}_{2}<60 \mathrm{~mm} \mathrm{Hg}$ despite $\mathrm{O}_{2}$ supplementation, or (6) bilateral lobar pneumonia. The co-morbid conditions included chronic obstructive airway disease, bronchiectasis, chronic renal failure, diabetes mellitus, heart failure or immunosuppression. The category of moderate pneumonia in our classification corresponds to the same category as patients who need hospitalisation but do not satisfy the criteria for severe pneumonia in the American Thoracic Society guidelines [6].

\section{Microbiological Techniques}

A positive sputum culture was defined as the identification of a single predominant organism in the sputum with a compatible gram stain and a positive cold agglutinin was defined as a titre of 1:32 using the quantitative dilution method. There were only 2 patients who had bronchoscopy done to obtain bronchial lavage sample for microbiological analysis. This reflects the practice in Kuwait where bronchoscopy is done only for immunocompromised patients with pneumonia or in patients with non-resolving pneumonia.

\section{Results}

\section{Microbial Aetiology}

There was 290 patients who met the inclusion criteria for the study, of which $57 \%$ were males, with mean age $41 \pm 19$ years. The contributions of the three centres to the study were as follows: Adan Hospital, 142 cases (49\%); Farwaniya Hospital, 81 cases $(28 \%)$, and Amiri Hospital, 67 cases (23\%). The distribution of cases according to severity was as follows: $27.5 \%$ mild cases, $61 \%$ moderate and $11.5 \%$ severe cases. The microbial aetiology 
Fig. 1. The percentages of the diagnostic yield of the different tests in the three groups. The only significant differences were in the positive yield from blood culture between the mild and severe groups $(* p=0.02)$ and between the moderate and severe groups $(* * \mathrm{p}=$ 0.02 ).

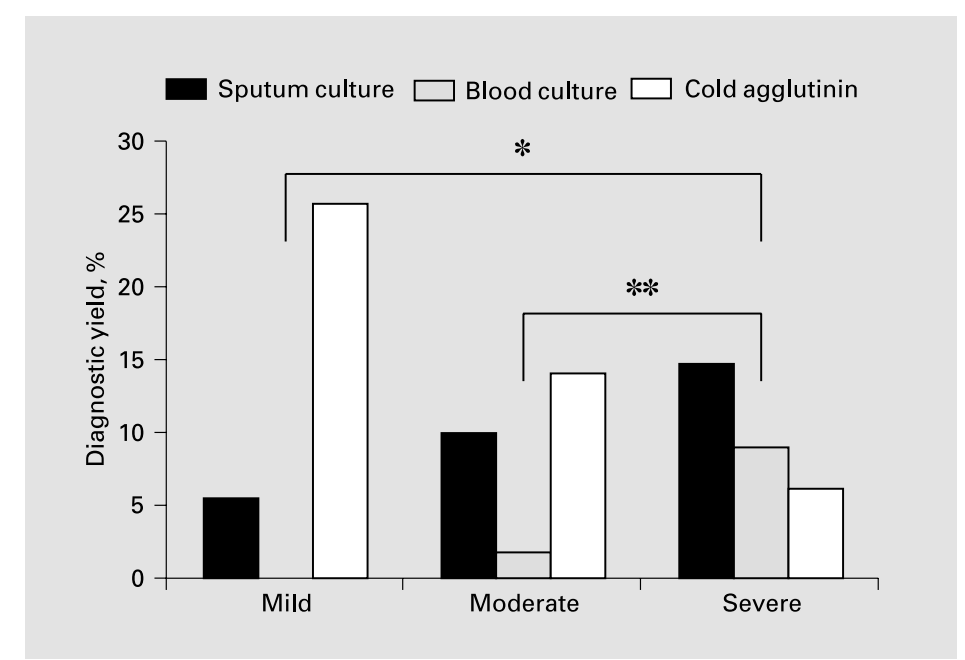

of pneumonia was identified in 44 cases only (15\% of the total cases): $11.5 \%$ in the mild cases, $14 \%$ in the moderate cases and $22.5 \%$ in the severe cases. The differences between the groups in terms of the rate of identification of the causative organisms did not reach statistical significance. The most common organisms identified were Streptococcus pneumoniae 32\%, Mycoplasma pneumoniae 20\%, Haemophilus influenzae 14\%, Mycobacterium tuberculosis $11 \%$, gram-negative organisms other than Haemophilus species 11\% and others $12 \%$. S. pneumoniae was the leading identified organism in each of the 3 years studied. The diagnosis of M. pneumoniae was based on clinical suspicion, a positive cold agglutinin test and lack of other microbial aetiology rather than definite serological diagnosis. The most common tests performed for the identification of the microbial organisms were sputum culture: 260 tests with $24(9 \%)$ positive tests, blood culture: 269 tests with 6 (2\%) positive tests, and cold agglutinin: 176 tests with $32(18 \%)$ positive tests. The diagnostic yields of both sputum and blood cul- tures increased as the severity of the pneumonia increased while the diagnostic yield of cold agglutinins decreased as the severity of pneumonia increased (fig. 1).

\section{Treatment}

The initial empirical antibiotics given in the first $48 \mathrm{~h}$ of admission were considered for this analysis. There were 244 patients (84\%) who received a single antibiotic as initial therapy while 46 patients (16\%) received a combined antibiotic regimen. The most common classes of antibiotics used are presented in table 1. A macrolide was the most common class of antibiotic used for the mild and moderate cases while a combined regimen of antibiotic, which invariably included third-generation cephalosporin and an aminoglycoside, was usually for severe cases (table 1).

\section{Outcome}

There were 8 patients who were admitted to the intensive care unit and 3 patients ( $1 \%$ of the total cases) died. 
Table 1. The most common antibiotics used in the treatment of CAP according to the severity

\begin{tabular}{|c|c|c|c|c|}
\hline $\begin{array}{l}\text { Severity of } \\
\text { pneumonia }\end{array}$ & First drug & Second drug & Third drug & Fourth drug \\
\hline Mild & macrolide (44\%) & ampicillin (34\%) & tetracycline $(7.5 \%)$ & penicillin $(5 \%)$ \\
\hline Moderate & ampicillin (24\%) & macrolide $(23 \%)$ & combined $^{1}(15 \%)$ & $\begin{array}{l}\text { third-generation } \\
\text { cephalosporin }(15 \%)\end{array}$ \\
\hline Severe & combined $^{2}(47 \%)$ & $\begin{array}{l}\text { third-generation } \\
\text { cephalosporin }(19 \%)\end{array}$ & penicillin $(12 \%)$ & $\begin{array}{l}\text { macrolide or } \\
\text { ampicillin }(9 \%)\end{array}$ \\
\hline
\end{tabular}

The numbers in parentheses indicate the percentage of patients who received this treatment among all patients in that severity group.

1 The most common combination for the moderate group was third-generation cephalosporin and erythromy$\operatorname{cin} 37 \%$.

2 The most common combination for the severe group is third-generation cephalosporin and aminoglycoside $60 \%$.

\section{Discussion}

This is the first study on CAP among adults in Kuwait. It shows that there are a large percentage of patients with mild CAP admitted to the hospital. The rate of identification of microbial aetiology is poor especially in mild cases.

A number of prospective studies investigating the aetiology of CAP in hospitalised patients have recently been published [2, 79]. Despite extensive investigations including serological tests for atypical organisms and viruses, the microbial aetiology was not identified in $30-44 \%$ of the cases. There was only one study where the rate of identification of the microbial aetiology was very high at $97 \%$ [7]. Furthermore microbiological investigations in routine clinical practice have identified the microbial aetiology in only $26 \%$ of patients hospitalised with CAP [10]. In a prospective study of patients with CAP treated in the community the rate of identification of microbial aetiology was 55\% [11]. The retrospective nature of our study, incomplete sam- ple collection and lack of specific tests for atypical organisms or viruses explain the low rate of identification of the microbial aetiology. Despite these limitations, our study showed that $S$. pneumoniae was the leading causative organism in CAP similar to most of the other published studies [2, 7, 9]. This is the first report on CAP among adults in $\mathrm{Ku}$ wait, hence we cannot compare our results to previous studies. In a prospective study of acute lower respiratory tract infection among children in Kuwait [1], the most common causative organisms of pneumoniae were: respiratory syncytial virus $29 \%$, influenza A and B viruses $12 \%, H$. influenzae type b $8 \%$ and S. pneumoniae $6 \%$.

The relationship between the severity of CAP and the rate of identification of the microbial aetiology has not been studied systematically. However the rate of identification of the microbial aetiology in patients with mild CAP treated in the community [11] was lower than that in hospitalised patients with more severe CAP. In our study, there was a trend of higher rate of identification of micro- 
bial aetiology in the moderate and severe groups compared to the mild group. This difference did not reach statistical significance, probably due to the small number of patients in each group.

The diagnostic yield from blood culture was very poor in our study. None of the patients in the mild group had a positive blood culture and only 3 patients out of 163 (2\%) in the moderate group had positive blood cultures while 3 patients out of 32 (9\%) had positive blood cultures in the severe group ( $p=0.02$ for the difference between the mild and the severe groups). Our results are similar to previously published studies, which showed poor clinical utility of blood culture in CAP [12, 13]. The value of sputum Gram stain to determine the microbial aetiology of CAP is controversial. It has been shown that sputum Gram stain is useful in the initial evaluation of CAP when proper sample collection and examination are done [14]. However, its value under routine clinical practice has been debated [6]. In our study the diagnostic yield of sputum Gram stain and culture was only $9 \%$. We do not have data on the quality of sputum samples that were submitted, which limits our ability to interpret the findings.

The high percentage of mild patients (27\%) of total study population) who were admitted to the hospital is surprising. We used strict criteria to define the mild cases. These criteria included age $<60$ years, absence of co-morbid conditions, absence of high fever $\geq 38.3^{\circ} \mathrm{C}$, lack of any clinical or radiological signs indicative of severe disease. The above factors have been shown to predict the need for hospitalisation in CAP [15]. Increasing the awareness of these criteria will help to reduce admission rate and hence costs associated with CAP. Although the antibiotics used (table 1) for the treatment of CAP did not strictly follow internationally recommended guide- lines [4-6], the outcome of the patients was very good. The major deviations from the guidelines were: (1) ampicillin was used frequently for mild CAP while the guidelines recommend using a macrolide, (2) the guidelines recommend to use either second- or third-generation cephalosporin \pm macrolide for moderate CAP while $47 \%$ of the patients in the moderate group received either ampicillin or macrolide alone, and (3) the guidelines recommend a combination of high dose erythromycin and third-generation cephalosporin for the severe group while most of the patients in the study received either single agent or a combination of third-generation cephalosporin and aminoglycoside. We feel that a prospective study is needed to determine the wide spectrum of microbial aetiologies in CAP before local guidelines can be formulated. The frequent use of aminoglycosidecontaining regimens in severe pneumonia cannot be explained. The use of aminoglycosides is only recommended for patients with structural lung disease where Pseudomonas organisms are suspected [16].

The most significant findings of this study are: (1) the identification of the causative organisms in CAP is poor under routine clinical practice conditions; (2) there is a relatively large percentage of patients with mild CAP admitted to the hospital who probably could have been safely treated in the community, and (3) there is clearly a need for a prospective study to determine the spectrum of the microbial aetiologies in CAP. This will provide data to formulate local guidelines for the treatment of CAP.

\section{Acknowledgement}

We would like to thank Prof. T.D. Chugh and Prof. A.S. Mustafa for reviewing the manuscript. 


\section{References}

1 Hijazi Z, Pacsa A, el-Gharbawi F, Chugh TD, Essa S, el-Shazli A, Abd el-Salam R: Acute lower respiratory tract infections in children in $\mathrm{Ku}-$ wait. Ann Trop Paediatr 1997;17: 127-134.

2 Fang G-D, Fine M, Orloff J, Arisumi D, Yu VL, Kapoor W, Grayston T, Wang SP, Kohler R, Muder RR, Yee YC, Rihs JD, Vickers RM: New and emerging aetiologies for community acquired pneumonia with implications for therapy. Medicine (Baltimore) 1990;69:307-316.

3 Marrie TJ: Community-acquired pneumonia: State of the art article. Clin Infect Dis 1994;18:501-513.

4 Mandell LA, Niederman M, the Canadian Community Acquired Pneumonia Consensus Conference Group: Antimicrobial treatment of community acquired pneumonia in adults: A conference report. Can J Infect Dis 1993;4:25-28.

5 The British Thoracic Society: Guidelines for the management of community acquired pneumonia in adults admitted to hospital. $\mathrm{Br}$ Hosp Med 1993;49:346-350.
6 American Thoracic Society: Guidelines for the initial management of adults with community acquired pneumonia: Diagnosis, assessment of severity, and initial antimicrobial therapy. Am Rev Respir Dis 1993; 148:1418-1426.

7 MacFarlane JT, Finch RG, Ward MJ, Macrae AD: Hospital study of adult community-acquired pneumonia. Lancet 1982;ii:255-258.

8 Marrie TJ, Durant $\mathrm{H}$, Yates L: Community acquired pneumonia requiring hospitalisation: 5 year prospective study. Rev Infect Dis 1989;11:586-599.

9 Neil AM, Martin IR, Weir R, Anderson R, Chereshsky A, Epton MJ, Jackson R, Schousboe M, Frampton C, Hutton S, Chambers ST, Town GI: Community acquired pneumonia: Aetiology and usefulness of severity criteria on admission. Thorax 1996;51:1010-1016.

10 Woodhead MA, Arrowsmith J, Chamberlain-Webber R, Wooding $\mathrm{S}$, Williams I: The value of routine microbial investigation in community-acquired pneumonia. Respir Med 1991;85:313-317.
11 Woodhead MA, Macfarlane JT, McCracken JS, Rose DJ, Finch RG: Prospective study of the aetiology and outcome of pneumonia in the community. Lancet 1987;i:671674.

12 Ostergaard L, Andersen PL: Aetiology of community-acquired pneumonia: Evaluation by transtracheal aspiration, blood culture, or serology. Chest 1993;104:1400-1407.

13 Chalasani NP, Valdecanas ML, Gopal AK, McGowan JE, Jurado RL: Clinical utility of blood cultures in adult patients with community-acquired pneumonia without defined underlying risks. Chest 1995;108: 932-936.

14 Boerner DF, Zwadyk P: The value of the sputum Gram's stain in community-acquired pneumonia. JAMA 1982;247:642-645.

15 Fine MJ, Smith DN, Singer DE: Hospitalisation decision in patients with community-acquired pneumonia: A prospective cohort study. Am J Med 1990;89:713-721.

16 Bartlett JG, Breiman RF, Mandell LA, File TM Jr: Community-acquired pneumonia in adults: Guidelines for management. Clin Infect Dis 1998;26:811-838. 